\title{
Aporte del Departamento de Orientación al desarrollo del liderazgo pedagógico: un estudio desde la opinión de directores de Institutos de Enseñanza Secundaria en Andalucía ${ }^{1}$
}

Contribuição do departamento de
orientação para o desenvolvimento da
liderança educacional: um estudo do ponto
de vista dos diretores dos institutos de
educação secundária na Andaluzia

Contribution of the Guidance Department to the pedagogical leadership development: a study on the opinion of the high school directors in Andalusia

\author{
Jesús Domingo Segovia ${ }^{2}$ \\ Maximiliano Ritacco Real ${ }^{3}$
}

\section{RESUMEN}

El estudio forma parte de un proyecto de investigación sobre liderazgo centrado en el aprendizaje en Secundaria. Responde a la necesidad de

DOI: $10.1590 / 0104-4060.43547$

1 El estudio ha sido subvencionado por el Ministerio de Ciencia y Tecnología (España) dentro del VI Plan Nacional de Investigación Científica (Ref. EDU2010-16131), Desarrollo e Innovación Tecnológica (I+D+I).

2 Universidad de Granada. Facultad de Ciencias de la Educación. Granada, España. Campus de Cartuja, s/n. Código Postal: 18071.E-mail: jdomingo@ugr.es

3 Universidad de Zaragoza, Departamento de Educación. Teruel, España. Calle Atarazana, 4. Código Postal: 44003. E-mail: ritacco@unizar.es 
apoyar el desarrollo del liderazgo pedagógico de la dirección escolar en Andalucía. Busca comprender, desde la teoría fundamentada de estos profesionales y el análisis de su discurso, en qué medida encuentran apoyo en los departamentos de orientación, en qué ámbitos y qué necesidades plantean al respecto. Recoge evidencias de 15 entrevistas en profundidad a directivos escolares con una trayectoria de mejora. Concluye que el Departamento de Orientación, junto a la jefatura de estudios, son los principales soportes al liderazgo educativo y distribuido, conformando su grupo motor de mejora. Pero depende de los profesionales, centros y contextos. Es más evidente en contextos vulnerables y con "buenos" orientadores y directores que han redireccionado sus funciones hacia agentes de apoyo para la mejora educativa y el asesoramiento como colega crítico.

Palabras clave: dirección escolar; liderazgo pedagógico; orientación educativa; apoyo; educación Secundaria.

\title{
RESUMO
}

O estudo faz parte de um projeto de pesquisa sobre liderança com foco na aprendizagem na educação secundária. Responde à necessidade de apoiar o desenvolvimento da liderança pedagógica da gestão escolar na Andaluzia. Procura entender, a partir da teoria fundamentada desses profissionais e da análise de seu discurso, em que medida eles encontram apoio nos serviços de orientação, em que âmbitos e quais necessidades possuem. A pesquisa recolheu informações de 15 entrevistas feitas a diretores de escolas com uma trajetória de melhoria. Concluiu que o Departamento de Orientação juntamente com o líder de estudos são os principais suportes da liderança educacional, formando um grupo motor de melhoria. Mas isso depende dos profissionais, instituições e contextos. Ele é mais evidente em contextos vulneráveis e com "bons" orientadores e diretores que redirecionam suas funções para a de agentes de apoio para melhorar a educação e a orientação como colega crítico.

Palavras-chave: direção escolar; liderança pedagógica; orientação educacional; apoio; educação secundária.

\begin{abstract}
This study is part of a research project about leadership focused on learning at high school. It responds to the need to support the development of educational leadership of the school management in Andalusia. We search to understand, from the grounded theory of these professionals and the analysis of their speech, to what extent they find support in the guidance department, in which fields, and what needs they consider in this respect. We collected evidence
\end{abstract}


of 15 in-depth interviews to school principals with an improving career. The conclusion was that those guidance departments, along with the head of studies, are the main educational supports and their distributed leadership gathers a group that is shaping its power of improvement. But it is up to professionals, schools and contexts. It is more evident in vulnerable contexts and with "good" school counselors and school principals that have redirected their functions to be supporting agents for educational improvement and better advice as a critical colleague.

Keywords: school management; educational leadership; educational guidance; support; secondary education.

\section{Introducción}

El estudio que se presenta forma parte del proyecto de investigación titulado "Liderazgo centrado en el aprendizaje y su impacto en la mejora: Prácticas y resultados en Secundaria", subvencionado por el Ministerio de Ciencia y Tecnología dentro del VI Plan Nacional de Investigación Científica, Desarrollo e Innovación Tecnológica (I+D+I), se integra en la "Red de Investigación sobre Liderazgo y Mejora Educativa" (RILME) y se adjunta a las recomendaciones del Informe "Improving School Leadership" de la Organization of economic cooperation and development (OECD) (2009).

A nivel general se ha utilizado una metodología de estudio multicaso (multicase study) recogiendo evidencias de un amplio rango de técnicas de investigación, tanto de corte cuantitativo como cualitativo (mixed methods), al servicio de una mejor comprensión de los efectos del liderazgo en la Comunidad Autónoma de Andalucía.

Dentro de este marco, el estudio centra su interés en uno de los objetivos generales del proyecto: analizar y comprender qué buenas prácticas de liderazgo en los Institutos de Secundaria, no limitadas al equipo directivo, contribuyen decididamente a la mejora de la educación. Y más particularmente comprender, de una parte, cómo es y cómo se desarrolla el apoyo al desarrollo del liderazgo para el aprendizaje en los centros de educación secundaria en Andalucía, y, de otra, cómo es percibida esta acción por los directivos escolares.

La investigación educativa actual viene evidenciado que la mejora escolar se juega a nivel de cada centro escolar - entre otras claves - en función de cómo se genere y utilice su capacidad de mejora de manera sostenible en el tiempo. En ello, el liderazgo educativo es percibido como un elemento clave para generar mejora desde abajo, creando condiciones institucionales que hagan 
más eficaces las organizaciones escolares. (BOLÍVAR; LÓPEZ; MURILLO, 2013). Existe suficiente evidencia empírica sobre liderazgo para el aprendizaje y su impacto en los resultados (LEITHWOOD, 2009) que lo señala como clave para desarrollar y dinamizar "lo pedagógico" y las interacciones efectivas entre profesorado, alumnado y currículum. (ELMORE, 2010).

Parece fundamental, pues, una nueva comprensión de la dirección escolar, entendida como liderazgo educativo (BOLÍVAR, 2012; LEITHWOOD, 2009) cada vez más centrado hacia el núcleo pedagógico (ELMORE, 2010) y con capacidad para influir tanto en los resultados de aprendizaje como en los procesos de desarrollo del "capital profesional" (HARGREAVES; FULLAN, 2012) de sus centros. No en vano, lo que define en buena medida a los buenos o malos directores es el tiempo y los esfuerzos centrados en tareas pedagógicas. Y en ello se necesita una "conexión crítica" entre este liderazgo para el aprendizaje, la cooperación profesional y la emergencia de un liderazgo distribuido (SPILLANE; CAMBURN; PAREJA, 2009; SPILLANE, 2006) en el seno de una comunidad. (SPILLANE, 2006). Muy especialmente cuando existe una perspectiva inclusiva del liderazgo y la mejora en y para la justicia social. (MURILLO; KRICHESKY; CASTRO; HERNÁNDEZ-CASTILLA, 2010; WALKER, 2006).

Pero, conviene no olvidar en este punto que la dirección escolar en España no está profesionalizada, y como ha denunciado el Informe TALIS (OECD, $2009,2013)$ y otros estudios (GAGO, 2006), el liderazgo educativo entre los directores escolares españoles es una función claramente disminuida que necesita apoyo para ser recompuesta. Se necesita pues apoyo y asesoría, funciones que bien pueden desarrollar los profesionales de la orientación, como comentan Domingo, Caballero y Barrero (2013):

\footnotetext{
Las razones son obvias. De una parte, tienen una presencia real consolidada en todos los centros de Educación Secundaria [...] y de otra, su formación psicopedagógica y el rol que marca la norma al delimitar sus funciones y ámbito de trabajo, les confiere una especial visión sistémica y educativa [...] una posición estratégica nada despreciable. (DOMINGO; CABALLERO; BARRERO, 2013, p. 3).
}

Asentada la figura del orientador escolar, tras más de dos décadas de presencia real en los centros de educación secundaria, junto a sus funciones técnicas, están emergiendo otras más impactantes, con una misión por el cambio (ASCA, 2008; BOZA; TOSCANO; SALAS, 2007), e incluso como líder 
(FORD; NELSON, 2007) comprometido con la mejora y el buen aprendizaje para todos. (MARTÍNEZ; KRICHESKY; GARCIA, 2010). Aunque esta realidad está lejos aún de poder considerarse conseguida. (AMATEA; CLARK, 2005).

Así las cosas, esta figura profesional podría quedar ahora situada en una posición estratégica, que al tiempo de trabajar muy apegado al aula, posee un amplio recorrido y visión sistémica del centro (LAGO; ONRUBIA, 2011), habilitándole especialmente para el trabajo colaborativo con el profesorado y el equipo directivo. (JANSON; STONE; CLARK, 2009). Por lo cual, en determinadas circunstancias y desde un rol de "acompañamiento crítico profesional" (DOMINGO, 2009; GURR; HUERTA, 2013; SWAFFIELD, 2004), pueden ser especialmente bienvenidos estos determinados apoyos para reforzar y operativizar el aprendizaje de toda la comunidad y la cooperación mutua. (BOLAM et al., 2005). Todo en la línea de ir conformando un equipo de liderazgo, o grupo motor, por la mejora y la justicia. (WALKER, 2006). Pero también, no pocas veces, queda la posibilidad de que puedan ser percibidas sus acciones, como injerencias poco pertinentes y que entran en fricción con el propio desarrollo del liderazgo del director y la línea de mejora emprendida por el centro. Y en ello, para bien o para mal, intervienen mucho las percepciones y los diferentes juegos de interacción que se producen entre los orientadores y los directivos. (KIRCHNER; SETCHFIELD, 2001).

Para la comprensión de esta situación y como se entremezclan perspectivas, identidades, historias de vida, cultura profesional e institucional, y otra serie de circunstancias que tejen escenarios complejos a favor o en contra de este encuentro profesional, este estudio recurre a relatos de experiencias de vida profesional de directivos, narrados en primera persona, que alumbren realidades particulares de interrelación profesional entre orientadores y directivos escolares.

\section{Metodología}

\section{Diseño y fundamento metodológico}

Para estudiar la comprensión de la percepción del grado de apoyo e influencia de la labor de la orientación educativa en el desarrollo de un liderazgo pedagógico en los centros escolares, se utiliza una metodología de investigación de corte cualitativo. (FLICK, 2007). La interrelación profesional como objeto de estudio es resultado de una realidad social en un contexto dado y es inseparable de los propios sujetos intervinientes, de sus expectativas, intenciones y sistemas 
de valores. Construcción (social) que se apoya en los significados compartidos (comunicación e interacción) contextualizados en una cultura, una identidad profesional, unas particulares relaciones profesionales y la propia arquitectura de conocimiento profesional fruto de la experiencia reflexionada.

Se aborda este conocimiento desde el análisis del discurso y de las percepciones de los sujetos participantes que nacen de la construcción biográfica de una teoría fundamentada emergente de su práctica profesional. (WERTZ; CHARMAZ; MCMULLEN, 2011). Y todo ello sin violentar los principios básicos de sistematización y procedimientos de investigación propios de la teoría fundamentada (ground theory). (CHARMAZ, 2004; STRAUSS; CORBIN, 2002). Se identifican las unidades de significado de los propios relatos, tras observar regularidades en las unidades de registro (UR) después de reiterados usos y preguntas sucesivas sobre el tema. Se continúa el proceso con la sistematización y categorización de las UR por similitudes o valores compartidos que aportan la "base" para la creación de teoría. Va emergiendo y se va generando conocimiento sobre la posible influencia de la labor del Departamento de Orientación en el desarrollo de un liderazgo pedagógico en los centros públicos investigados. Todo ello desde la voz, las creencias, las ideas y las valoraciones acerca de las diferentes capacidades que tienen que desarrollar y que están integradas en el liderazgo pedagógico según estos directores y directoras.

\section{Criterios de selección y distribución de los informantes clave}

Se buscó un conjunto de institutos y directivos escolares como informantes clave pues contaban con un amplio recorrido en innovación o como líderes en procesos de mejora. Desde sus relatos de experiencia profesional y su conocimiento sustantivo, se podrían así extraer claves de reflexión desde las que repensar la práctica asesora de las y los profesionales de la orientación a la hora de apoyar el liderazgo pedagógico y promover la mejora de los resultados escolares de los estudiantes.

Se configuró una muestra intencional relativamente homogénea-restringida en función de tres criterios fundamentales: 1) de conveniencia, para garantizar la accesibilidad y predisposición a participar en la investigación; 2) contextual, para captar la perspectiva meso y micro de la labor de la dirección escolar en centros ubicados en contextos diferentes; y 3) una perspectiva (biográfica) cronológica y procesual de la tarea de dirección, para lo que se considera imprescindible que los sujetos entrevistados hayan estado en la dirección a lo largo de un período mínimo de 3 años.

Fueron seleccionados 15 centros de educación secundaria en la provincia de Granada (Andalucía) con los siguientes criterios: a) ubicación geográfica 
(cinco en el centro urbano, cinco en barrios aledaños al macro-centro y cinco en zonas periféricas y comarcales); b) indicadores de clase social y origen socio económico de sus estudiantes (cinco de clase media-alta, cinco de clase media y cinco de clase baja); c) oferta educativa de los centros (planes y programas de atención); y c) financiados con fondos públicos. Estos aspectos se pueden observar en la siguiente Tabla 1:

TABLA 1 - CRITERIOS DE SELECCIÓN Y DISTRIBUCIÓN DE LA MUESTRA DE CENTROS

\begin{tabular}{|c|c|c|c|c|}
\hline $\begin{array}{c}\text { Centros } \\
\text { Educativos } \\
\text { (investigados) }\end{array}$ & $\begin{array}{c}\text { Ubicación y } \\
\text { contexto geográfico }\end{array}$ & $\begin{array}{l}\text { Composición y } \\
\text { clase social* }^{*}\end{array}$ & $\begin{array}{c}\text { Oferta educativa de los } \\
\text { centros }\end{array}$ & Tipo de centro \\
\hline $\mathrm{C} 1$ al C5 & $\begin{array}{c}\text { Zonas periféricas y } \\
\text { comarcales }\end{array}$ & $\begin{array}{l}\text { Clase Baja o } \\
\text { Media-Baja }\end{array}$ & \multirow{3}{*}{$\begin{array}{c}\text { ESO, Bachillerato, } \\
\text { Programas de atención a la } \\
\text { diversidad, Programas de } \\
\text { Cualificación Profesional } \\
\text { Inicial (PCPI) }\end{array}$} & \multirow{3}{*}{$\begin{array}{l}\text { Financiado con } \\
\text { fondos Públicos }\end{array}$} \\
\hline $\mathrm{C} 6$ al C10 & $\begin{array}{l}\text { Macro-Centro } \\
\text { urbano }\end{array}$ & $\begin{array}{l}\text { Clase Media- } \\
\text { Baja }\end{array}$ & & \\
\hline $\mathrm{C} 11$ al C15 & Centro Urbano & Clase Media & & \\
\hline \multicolumn{5}{|c|}{ Nomenclatura de codificación de entrevista: $\mathrm{Cn}^{\circ}=$ Centro y número identificativo; $\mathrm{D}=$ Directores/as. } \\
\hline \multicolumn{5}{|c|}{ * Datos facilitados por los centros escolares y la administración educativa (Séneca) } \\
\hline \multicolumn{5}{|c|}{ Los casos $\mathrm{C} 5, \mathrm{C} 8, \mathrm{C} 11$ y $\mathrm{C} 12$ son mujeres, mientras que en el resto son varones } \\
\hline
\end{tabular}

FUENTE: Elaboración propia (2015).

\section{Técnicas de recogida y procesamiento de datos}

Se realizaron 15 entrevistas biográficas en profundidad, no estructuradas, tematizadas en los procesos de interacción profesional entre directivos y otros profesionales del centro educativo, entre los que se encontraban los orientadores.

El tema-guión de la entrevista indagaba sobre qué principios y elementos guiaban el desarrollo de su liderazgo, al tiempo que buscaba extraer claves comprensivas sobre cómo se apoya esta función desde labores pedagógicas y/o distribuidas. Se debatió sobre el desarrollo de las funciones en sí, así como en el tipo de intervención (grado de presencia, relevancia y apoyo) ya sea en primera persona o por asignación del directivo al orientador.

El tiempo total de las entrevistas $=18$ horas, 15 minutos (Media por entrevista $=1$ hora, 13 minutos). Edad de los entrevistados: Entre 63 y 37 años de edad (media de 50 años de edad), Género de los entrevistados $=4$ Mujeres y 11 Hombres.

Se utilizó el Software para el análisis de datos NVivo8 para organizar y analizar información, compararla y darle sentido de forma más rápida y segura. 
El procedimiento de análisis y categorización de los datos

La reducción de los datos, entendida como la síntesis y categorización de la información, se realizó inductivamente a partir del análisis de contenido, la secuenciación de preguntas y la profundización sucesiva, técnicas propias de la investigación sobre teoría fundamentada. (CHARMAZ, 2004; STRAUSS; CORBIN, 2002). Se completó este proceso con otras indagaciones e interpretaciones emergentes del análisis de discurso. (WERTZ et al., 2011).

La categorización y estructuración de los datos (Tabla 2 se inició con el análisis de la información recogida en el trabajo de campo. Con la ayuda del programa Nvivo8, los datos fueron extrayéndose de las fuentes de información y organizándose en unidades de registro (UR). De forma sucesiva, las UR se reconvirtieron en indicadores (I) al someterse a un " $1^{\circ}$ vuelco" y conformar un conjunto de pre-categorías. En la siguiente Tabla 2 se presenta, por centro y tipología de centro, el número de unidades de análisis (UA) y unidades de registro (UR) e indicadores (I) resultante de la primera fase del proceso de categorización. Al respecto, a priori a los resultados de la investigación, se observa la tendencia menor clase de centro mayor cantidad de indicadores que hacen referencia al liderazgo pedagógico, más concretamente y con mayor grado de presencia en los contextos con mayor riesgo de exclusión/fracaso.

TABLA 2 - CLASIFICACIÓN POR TIPO DE CENTRO DE UNIDADES DEANÁLISIS (UA) Y UNIDADES DE REGISTRO (UR) E INDICADORES (I) RESULTANTES DEL PROCESO DE CATEGORIZACIÓN

\begin{tabular}{|l|c|c|}
\hline \multicolumn{1}{|c|}{ Centro } & $\begin{array}{c}\text { Unidades de Análisis (UA) } \\
\text { (Párrafos analizados) }\end{array}$ & $\begin{array}{c}\text { Unidades de registro (UR) } \\
\text { Indicadores (I) }\end{array}$ \\
\hline Centro $1 ; 2 ; 3 ; 4 ; 5$ & $19 ; 17 ; 23 ; 17 ; 18$ & $107 ; 169 ; 133 ; 44 ; 67$ \\
\hline Total Clase Baja & 94 & 520 \\
\hline Centro 6,$7 ; 8 ; 9 ; 10$ & $28 ; 22 ; 18 ; 23 ; 23$ & $87 ; 153 ; 27 ; 76 ; 82$ \\
\hline Total Clase Media-Baja & 114 & 425 \\
\hline Centro $11 ; 12 ; 13 ; 14 ; 15$ & $22 ; 18 ; 18 ; 21 ; 17$ & $49 ; 64 ; 37 ; 57 ; 68$ \\
\hline Total Clase Media & 96 & 275 \\
\hline Total analizado & $\mathbf{3 0 4}$ UA & $\mathbf{1 2 2 0}$ UR - I \\
\hline
\end{tabular}

FUENTE: Elaboración propia (2015).

Continuando con la estructuración de los datos, luego del $1^{\circ}$ vuelco y con un bajo nivel de concreción, las pre-categorías emergentes pudieron ser organizadas en grupos. Posteriormente, un " $2^{\circ}$ vuelco" favoreció la reorganización de los indicadores en una serie de categorías ya consolidadas. En este punto, la información se presentaba de forma tal que emergía como una de las más referenciadas la categoría que evidenciaba la influencia (aporte) del Departamento de Orientación en el desarrollo del liderazgo pedagógico en los centros estudiados. 
TABLA 3 - FASES DEL PROCESO DE ESTRUCTURACIÓN SOMETIDAS ALANÁLISIS DE CONTENIDO

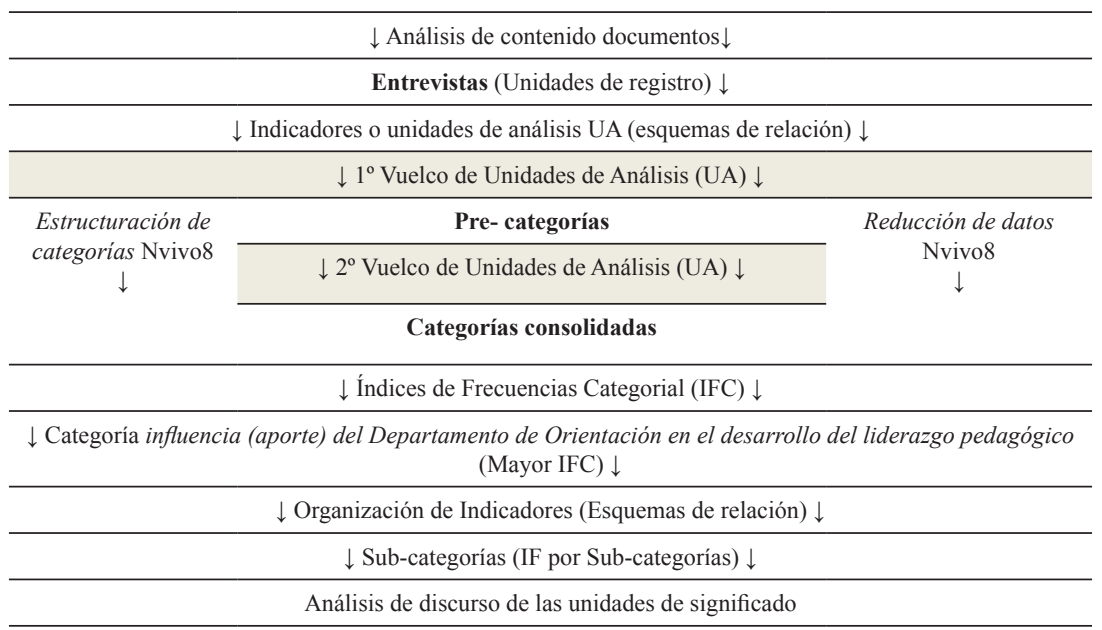

FUENTE: Elaboración propia (2015).

En la fase final, se aplicó un análisis por medio de esquemas de relación (con flechas, tipos de redes y nexos), el cual nos permitió observar la forma en que se predisponían sus indicadores (I). Agrupados significativamente en un conjunto de sub-categorías emergentes, su contenido hace referencia al aporte del Departamento de Orientación a la hora de desarrollar ciertas capacidades del liderazgo pedagógico en los centros:

*Desarrollo del programa y proyecto de centro y dirección / *Resolución de Conflictos / *Establecimiento de una organización colaborativa (entorno) / *Mejora de aprendizajes del alumnado / *Desarrollo de la formación del profesorado / *Establecimiento de líneas y directrices comunes / *Trabajo por competencias / *Desarrollo profesional (profesorado) / *Necesidades y limitaciones del departamento de orientación.

Una vez presentadas las sub-categorías emergentes de los relatos, se les asignó un índice de frecuencia categorial (IFC) otorgado por el número de indicadores (I) que contenían. Sin ser estadístico, sino aproximativo, el índice de frecuencia categorial (IFC) representa el peso significativo de la información contenida en las UR. Dicha frecuencia responde al porcentaje proporcional del IFC total de la categoría superior. 


\section{Resultados}

Primera categorización: verbalizaciones sobre el Departamento de Orientación

El análisis de datos, con las categorías emergentes, informa que existe una clara relación y vinculación entre la función de los profesionales de la orientación con el liderazgo escolar. Son múltiples las referencias que así lo demuestran. Entre ellas destacan algunas claves como elemento central del liderazgo, pilar del equipo motor de liderazgo, instigador de reflexión profesional, apoyo, mediador... (Ver Tabla 4).

Con el primer vuelco (Gráfico 1), se evidencia que la influencia e importancia del papel de la orientación se acentúa en los centros de clase baja, ya que más del $50 \%$ de la frecuencia total hace referencia a estos. En un segundo nivel de análisis no sólo es la cuestión de frecuencia porcentual, sino que además tiene una carga importante de contenido. En dichos contextos escolares se refiere más a un liderazgo de interrelaciones, de asesoría y de compromiso con el alumnado, mientras que en el resto de los centros es más una orientación de corte técnica, más centrada en otras funciones más tradicionales, pero de claro reconocimiento. (BOZA et al., 2007).

GRÁFICO 1 - INFLUENCIA DE LA ORIENTACIÓN POR TIPO DE CENTRO

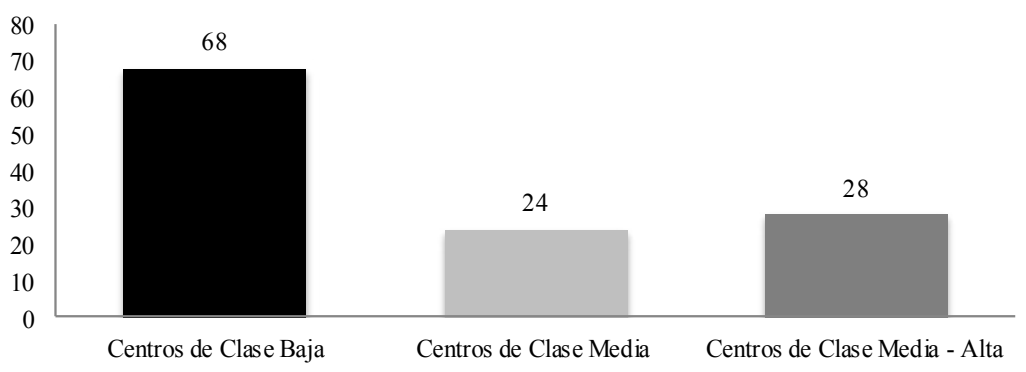

FUENTE: Elaboración propia (2015). 
TABLA 4 - VERBALIZACIONES DE DIRECTIVOS SOBRE EL DEPARTAMENTO DE ORIENTACIÓN

FR.16 [160] elemento central en la acción del Liderazgo Pedagógico. [161] motiva la reflexión del profesorado. [162] elemento central para el tratamiento de la convivencia. [163] aporta un gran potencial en formación y capacidad de trabajo. [164] respaldo con el alumnado. [165] con capacidad de desarrollo del proyecto de dirección y de centro [...] y liberan al director para realizar tareas administrativas [166] y asistenciales [...] MO.22 [126] Pilar dentro del equipo directivo (... aunque) no pertenece al equipo directivo. [128] ayuda en la organización escolar, aunque siempre que mantenga la misma línea y discurso con el equipo directivo. Y es [129] un aporte interno (para la diversidad de alumnado) y externo (como agente de enlace con otros agentes externos-redes). [130] y [126] necesario en una perspectiva global del centro [...] ET.10 [28] Capacidad de resolución de conflictos y [30] clave en un equipo didáctico (orientador, jefe de estudios, dirección). [25] apoyo para mejorar los aprendizajes, conocimiento de la normativa, adaptaciones curriculares, y nexo entre el equipo directivo y el profesorado [...] H.4 [25] Ayuda a mejorar los resultados de aprendizaje [...] A.16 [46] sirve para crear un clima de aprendizaje, buenos resultados y relación con la familia [...] A.17 [49] Capacidad de resolución de conflictos y asesoramiento del alumnado [...] BI. 12 [18] Capacidad para desarrollar el programa o proyecto educativo [...] BI. 11 [15] Apoyo en el proceso de resolución de conflictos y afrontar dificultades [...] JQ.12 [40] junto a la jefa de estudios, pilar de apoyo dentro del equipo directivo para organizar el centro y mejorar aprendizajes [...] JQ.13 [44] destaca su capacidad de coordinación - a la par del director [...] G.4 [9] Capacidad de establecer líneas comunes, si trabaja con el jefe de estudios, [10] buscar claves y convencimiento al profesorado cara a cara [...] AG.16 [38] Trabaja muchos ámbitos en los que el profesorado es reticente (y no siempre admite asesoramiento) [...] AG.12 [30] Puede incidir en el aprendizaje del alumnado. [31] mediadores con el profesorado - diplomacia del centro - autoridad indirecta [...] M12. [36] Capacidad resolución de conflictos, [37] gestión de recursos humanos y agente externos. [46] apoyo a la orientación

FUENTE: Elaboración propia (2015).

\section{Participación del Departamento de Orientación en las capacidades de liderazgo pedagógico}

En otro momento del análisis de la información, se reagruparon los indicadores de referencia y unidades de significado en función de las capacidades del liderazgo pedagógico. El Gráfico 2 destaca las dimensiones o capacidades del liderazgo que son reforzadas por las actuaciones del Departamento de Orientación. La influencia se evidencia en 9 de las 15 o 20 capacidades que puede integrar el desarrollo de un liderazgo pedagógico. (BOLÍVAR, 2012).

Según ella, la orientación está más presente en aquellas actuaciones que buscan: $1^{\circ}$ ) la mejora del aprendizaje de alumnado (crear un clima de aprendizaje 
en el centro, establecer niveles de convivencia mediante planes o programas); $2^{\circ}$ ) el apoyo en la resolución de conflictos (muy ligado a lo anterior y se refiere a conflictos alumnado-alumnado, alumnado-profesorado); $3^{\circ}$ ) El desarrollo del programa instruccional (el apoyo para la puesta en práctica y consecución de los objetivos del proyecto de centro y de dirección). No menos importantes, aunque menos referenciados son los dos siguientes: $4^{\circ}$ ) y $5^{\circ}$ ) El aporte de la orientación respecto a establecer e implementar unas líneas de actuación comunes (mantener unos ciertos niveles de cohesión en el centro mediante dinámicas conjuntas) y el establecimiento de lazos organizacionales con el contexto (ayuntamiento, servicios sociales, policía local, familia, etc.), cercanos a otras tareas de nexo o asesoría comunitaria. (RODRÍGUEZ, 2006).

GRÁFICO 2 - INFLUENCIA DEL DEPARTAMENTO DE ORIENTACIÓN EN LA DIRECCIÓN ESCOLAR. FRECUENCIA POR CAPACIDADES DEL LIDERAZGO PEDAGÓGICO

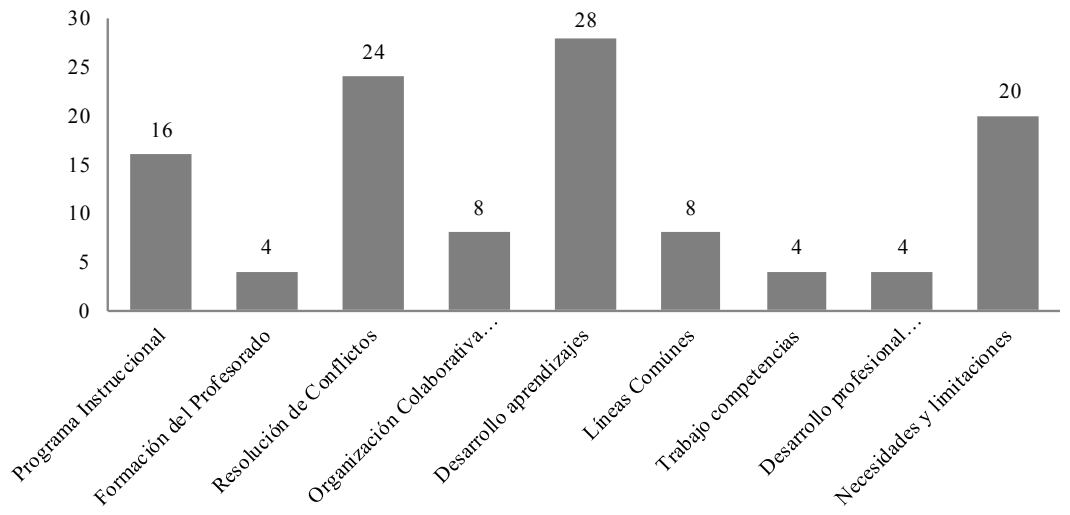

FUENTE: Elaboración propia (2015).

Da la sensación que gran parte del trabajo de la orientación respecto a la dirección escolar se focaliza en apoyar su tarea pedagógica y de gestión de programas o acciones, ya que - como denuncian los informes TALIS (OCDE, 2009, 2013) - la dirección escolar se encuentra bastante disminuida. 
GRÁFICO 3 - INFLUENCIA DEL DEPARTAMENTO DE ORIENTACIÓN EN LA DIRECCIÓN ESCOLAR. FRECUENCIA POR TIPO DE CENTRO Y CAPACIDADES DEL LIDERAZGO PEDAGÓGICO

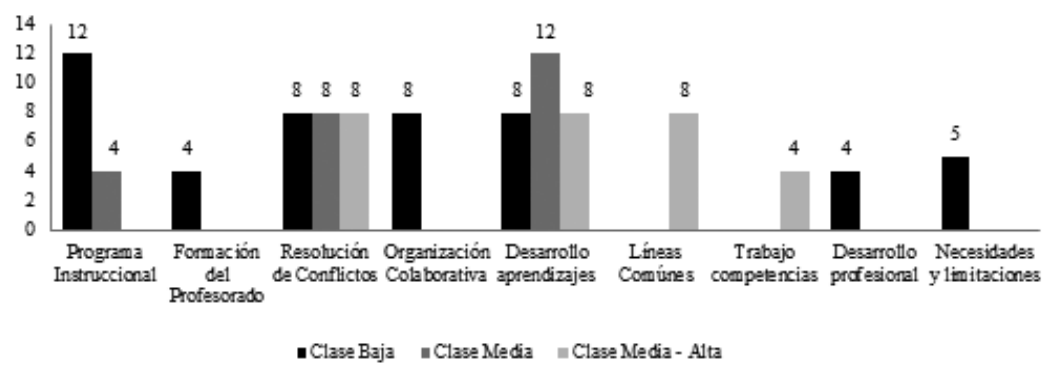

FUENTE: Elaboración propia (2015).

El Gráfico 3 esclarece un poco más el panorama. Se evidencia que la orientación no solo es más requerida en los centros de clase baja, sino que además sus directivos verbalizan que requieren de su intervención de forma distribuida en varias capacidades definitorias del liderazgo pedagógico, salvo en dos. Y muy especialmente en apoyo al programa instruccional. Mientras que los de clase media destacan el desarrollo de aprendizajes y en los contextos menos vulnerables solicitan apoyo para tener líneas comunes o trabajar las competencias. Ámbitos que no son verbalizados por el resto de directivos.

Otra cuestión es que el apoyo al director respecto a la resolución de conflictos y la mejora de los aprendizajes es algo que atañe a los departamentos de orientación de los tres tipos de centro. Lo que es muestra de su implantación y que son campos, escenarios y temáticas claramente asumidas por estos profesionales, lo que coincide con las indicaciones de la American School Counselor Association. (ASCA, 2008).

Una categoría emerge con fuerza y no responde directamente a las capacidades del liderazgo, aunque sí de forma indirecta, pues muestra claramente cómo funciona o debería funcionar esta figura profesional o su interrelación profesional e implicación en el proyecto educativo. Se refiere especialmente al testimonio de directivos escolares en contextos de vulnerabilidad en cuanto a necesidades y limitaciones del Departamento de Orientación. En ellas, se coincide con Swaffield (2004) y Walker (2006) en afirmar:

- El potencial como colega crítico de la orientación y en la necesidad de otorgarle reconocimiento como parte del equipo motor del centro educativo;

- Necesidad de que participen y apoyen una perspectiva global del centro (esta cuestión diferencia claramente a estos profesionales del resto del profesorado); 
- Debe ser un pilar dentro del equipo directivo y se reclama (especialmente a la Administración Educativa) que forme parte de la estructura oficial del equipo directivo, como apoyo fundamental al desarrollo de un proyecto educativo con capacidad de incidir en el éxito para todos y frente a la vulnerabilidad;

- Necesidad de que el Departamento de Orientación mantenga una misma línea y discurso con el equipo directivo. Esto demuestra que el apoyo del Departamento de Orientación es clave, en gran medida, para legitimar la falta de autoridad pedagógica del director a efectos del profesorado. Éste punto, según la investigación actual (KIRCHNER; SETCHFIELD, 2001), es el principal escoyo que suelen encontrar cuando alguno de los dos no comparte esta idea de co-liderazgo para el aprendizaje o percibe al otro como competidor;

- Debe ubicarse junto con el jefe de estudios, como un "caballo de batalla" del director y de la línea pedagógica del centro;

- Sin olvidar, como se ha destacado, su papel en el desarrollo profesional del profesorado. Lo que insiste en la línea reivindicativa de Zeichner (2010).

\section{Tercer nivel de profundización, análisis de discurso}

En un análisis más pormenorizado de las unidades de registro se detectan elementos discursivos dignos de ser tenidos en cuenta. A continuación, mediante el testimonio de los directivos, se describe cómo valoran el rol profesional de la orientación, en qué cuestiones participan y cómo es percibida su función de apoyo al liderazgo pedagógico.

Pasados aquellos tiempos de tener que ganarse un lugar y un reconocimiento, hoy están plenamente establecidos y reconocidos en los centros educativos:

la orientación en este centro es fundamental (FR. 16 [162]). Incluso, señalando que No es un profesor más del centro. Él tiene que tener una visión global del centro más aún que el profesorado! es como el director, coincidimos en eso, en la visión global del centro... (MO.22 [131]).

Se identifican claramente y se les asignan espacios profesionales y temáticos también específicos. Frases como las siguientes delatan esta situación, en la que reconocen su labor profesional con la diversidad: "Yo no había contemplado nunca a este alumnado" (BI. 12 [18]), con el desarrollo de capacidades 
y competencias, "fomentar la expresión oral" (H.4 [25]) o en situaciones de conflicto, "están cuando se presenta un conflicto" (ET. 10 [28]) y "tienen la técnica" [30], "ha generado un grupo de mediación" (A.17 [49])... y que es un campo tan necesario como necesitado de más presencia: "se necesitaría potenciar esta figura" (M.12 [36]); especialmente cuando se ve su utilidad en ámbitos hasta hace poco desconocidos como el trabajo con colegas: "están en contacto con el profesorado. Un buen orientador, es fundamental, a la hora de reflexionar con los compañeros sobre práctica docente, sobre programaciones, necesidades, autoevaluación..." (FR.16 [161]). Eso sí, no cualquier orientador, sino "un buen orientador"; con toda la carga de significado que comporta esta adjetivación.

Los directivos señalan también que los "buenos" orientadores son un elemento clave en su equipo y en sus proyectos. "Nuestro orientador fue una baza a favor" (BI. 12 [18]). "uno de mis pilares" (FR.16 [164]). Eso sí, siempre en un "grupo motor", junto a la jefatura de estudios (verdadero líder pedagógico en los centros educativos españoles), que complementan y operativizan la función directiva. Verbalizan contundentes en este sentido que:

[...] la orientación tiene que ser parte del equipo directivo por decreto [enfatizando aún más si cabe la necesidad]... aunque el problema es que no lo está... (MO. 22 [130]); [...] en la mejora del alumnado sobre todo, yo estoy muy tranquilo porque sé que la jefatura de estudios y el departamento de orientación funcionan muy bien (JQ.13 [44]); [...] generalmente las ideas parten de la directiva [dirección y jefatura de estudios], con la ayuda del departamento de orientación (JQ.12 [40]); Orientadora y jefe de estudio creo que son figuras básicas, son las dos manos de una dirección del centro. Es muy importante que, primero, el equipo directivo está unido, y segundo, que estas dos figuras estén activas a tu lado (AG.12 [31]); [...] me están siendo de una ayuda incalculable ya que están en el campo de batalla, ofertando digamos el primer cara a cara (AG.4 [10]).

Pero, aún pesa el estereotipo clínico, que aparece casi sin querer: "el psicólogo en el instituto" (M.12 [36]), "resuelven los problemas" (A.17 [15]), "es psicólogo educativo y ha sabido lidiar con ello" (BI. 12 [18]). Si bien son conscientes también de que este rol "se tiene que ampliar" (MO.22 [129]), hacia más participación en la vida del centro y las aulas, la comunidad y la integración en el equipo directivo y proyecto educativo. 
el aporte de la orientación en este centro está muy claro, es atención a la diversidad; pero también tiene que ampliar un poco a la atención a profesorado familias, instituciones... Tiene que participar en las relaciones del pueblo, más en un centro rodeado de zonas en riesgo. Incluso debiera ser parte del equipo directivo (MO.22 [129]).

Otros elementos del discurso devuelven el tema a la cruda realidad, pues muestran que todo depende del caso o del profesional, más que de la función o cargo. En este sentido destaca de forma contundente el constructo "suerte", frente al "tiene que" o "debiera": "He tenido la suerte de tener un jefe de estudios y una orientadora que han sido vitales" (AG.12 [30]); "En mis 8 años de dirección [en relación de quien ha ocupado estas funciones], he tenido una gran mala suerte y una gran buena suerte" (AG.4 [10]). Cuestión ésta a repensar, pues tal vez haya que hacer algo más por cambiar y profesionalizar, para generalizar más la idea que aquí se baraja de "buen profesional de la orientación".

\section{Discusión}

Las evidencias obtenidas permiten vislumbrar un escenario esperanzador de mutuo reconocimiento e interrelación profesional en el camino de reconstrucción de la cultura de los institutos hacia la mejora y los mejores resultados de aprendizaje. Son muchos los comentarios que apuntan en este sentido y las funciones o temáticas en las que tienen cabida estas interrelaciones. Lo que, sin duda, es interesante en un marco de secundaria, que históricamente se ha prestado a ser especialmente departamentalizado y academicista, necesitado de reconstrucción identitaria (BOLÍVAR; DOMINGO, 2006); y en el que la orientación ha ido cuajando también desde su espacio técnico. Los indicios apuntan que parece ir conquistando y redimensionando nuevas funciones de asesoramiento psicopedagógico (LAGO; ONRUBIA, 2011) en un escenario más abierto y paulatinamente más consciente de que hay que desarrollar un proyecto educativo interesante, en el que los profesionales con mayor perspectiva y amplitud de miras - directivos y otros colegas críticos (GURR; HUERTA, 2013) - deberían formar el grupo motor para posibilitar el tránsito hacia la mejora, "capitalizando profesionalmente" la institución (HARGREAVES; FULLAN, 2012) y el crecimiento de la comunidad profesional de aprendizaje desde la colaboración. 
Ahora bien, pese a esta primera inferencia optimista sobre la realidad, también cabría señalar que no fueron institutos cualquiera los seleccionados como objeto de estudio. Tenían una reconocida trayectoria de mejora. Luego conviene ajustar y atemperar estos comentarios, pues no siempre es así en el resto de los Instituto de Educación Secundaria (IES). Incluso, en éstos, aunque son múltiples las referencias a los orientadores y sus posibilidades, o en demandas hacia su labor, no son tampoco ni generalizadas, ni reiteradas. Basta fijarse en los porcentajes de aparición y los silencios implícitos en temáticas que podrían parecer obvias.

Todo parece indicar pues, y en ello se coincide con la investigación internacional promovida, entre otras instituciones, desde ASCA (2008) - (STONE; CLARK, 2001) -, que aunque se apunte prometedora la línea de trabajo de "apoyo al liderazgo pedagógico", está aún por conquistar. Se necesita ir poniendo recurrentemente en valor la capacidad y la funcionalidad de estos profesionales para facilitar el cambio educativo deseado, y muy especialmente en los contextos de vulnerabilidad. (WALKER, 2006). Para lo que convendría apostar seriamente por participar activamente en acciones de acompañamiento al profesorado y directivos en el desarrollo de procesos de mejora educativa (SINK, 2009; SANTANA, 2008), partiendo obviamente de los espacios ya reconocidos (convivencia, conflicto, apoyo al aprendizaje...), sin renunciar a ejercer y compartir, por qué no, liderazgo en estas determinadas parcelas y procesos. (FORD; NELSON, 2007). Más aún si, como ha evidenciado el estudio, ya se lo reconoce como un "pilar" necesario para la función directiva.

En definitiva, directivos escolares y orientadores, superando recelos y viejas identidades (AMATEA; CLARK, 2005), pueden ser y debieran caminar en línea de ir reconociéndose e implicándose como aliados y corresponsables de la buena dirección del cambio hacia la justicia y el éxito para todos. Así pues, el apoyo al desarrollo de un liderazgo pedagógico en los centros de secundaria se reconoce como un estimulante y prometedor camino por recorrer.

\section{REFERENCIAS}

AMATEA, E.; CLARK, M. A. Changing Schools, Changing Counselors: A Qualitative Study of School Administrators' Conceptions of the School Counselor's Role. Professional School Counseling Journal, v. 9, n. 1, p. 16-27, 2005.

ASCA. School Counselor Competencies. American School Counselor Association (ASCA), 2008. Disponible en: <http://www.schoolcounselor.org/files/SCCompetencies. pdf $>$. Acceso en: 18 abr. 2015. 
BOLAM, R.; MCMAHON, A.; STOLL, L.; THOMAS, S.; WALLACE, M. (Dirs.). Creating and sustaining effective professional learning communities. Bristol: University of Bristol y Department for Education and Skills, DfES. Research Report RR637, 2005. Disponible en: <http://www.dfes.gov.uk/research/data/uploadfiles/RR637.pdf>. Acceso en: 27 feb. 2015.

BOLÍVAR, A. Politicas actuales de mejora y liderazgo educativo. Archidona: Aljibe, 2012.

BOLÍVAR, A.; DOMINGO, J. The professional identity of secondary school teachers in Spain, crisis and reconstruction. Theory and Research in Education, v. 4, n. 3, p. 339-355, 2006.

BOLÍVAR, A.; LÓPEZ, J.; MURILLO, J. Liderazgo en las instituciones educativas. Una revisión de líneas de investigación. Revista Fuentes, v. 14, p. 15-60, 2013.

BOZA, A.; TOSCANO, M. O.; SALAS, M. ¿Qué es lo que hace un orientador?: Roles y funciones del orientador en educación secundaria. XXI. Revista de Educación, v. 9, p. 111-131, 2007. Disponible en: <http://www.uhu.es/publicaciones/ojs/index.php/xxi/ article/viewArticle/520>. Acceso en: 25 mar. 2015.

CHARMAZ, K. Grounded Theory: Objectivist and Constructivist Methods. En: EMERSON, R. (Ed.). Contemporary field research. Boston, MA: Little, Brown and Company, 2004. p. 109-126.

DENZIN, Y.; LINCOLN, S. (Eds.). Strategies of Qualitative Inquiry. Handbook of Qualitative Research. 2. ed. Thousand Oaks, CA: Sage, 2004. p. 509-535.

DOMINGO, J. Asesoría a la escuela para la mejora de la enseñanza y el aprendizaje. En: Vv. Aa. Desarrollo de la gestión educativa en México: Situación y perspectivas. México D.F.: UPN, 2009. p. 101-126.

DOMINGO, J.; CABALLERO, K.; BARRERO, B. Support for the leadership for learning in secondary education. The case of the school counselors in Spain. European Scientific Journal (ESJ), v. 9, n. 13, p. 1-15, 2013. Disponible en: <http://eujournal.org/index.php/ esj/article/view/1037/1071>. Acceso en: 03 abr. 2015.

ELMORE, R. F. Mejorando la escuela desde la sala de clases. Santiago de Chile: Fundación Chile, 2010.

FLICK. U. Introducción a la Investigación Cualitativa. España: Morata, 2007.

FORD, A. D.; NELSON, J. A. Secondary School Counselors as Educational Leaders: Shifting Perceptions of Leadership. Journal of School Counseling, v. 5, n. 19, p. 1-27, 2007. Disponible en: <http://jsc.montana.edu/articles/v5n19.pdf>. Acceso en: 05 abr. 2015.

GAGO, F. La dirección pedagógica en los Institutos de Enseñanza Secundaria. Un estudio sobre liderazgo educacional. Madrid: CIDE - Centro de Investigación y Documentación Educativa, Ministerio de Educación y Ciencia, 2006. 
GURR, D.; HUERTA, M. The Role of the Critical Friend in Leadership and School Improvement. Procedia-Social and Behavioral Sciences, n. 106, p. 3084-3090, 2013.

HARGREAVES, A.; FULLAN, M. Capital profesional. Madrid: Morata, 2014.

JANSON, C.; STONE, C.; CLARK, M. A. Stretching leadership: A distributed perspective for school counselor leader. Professional School Counseling, v. 13, n. 2, p. 98-106, 2009.

KIRCHNER, G. L.; SETCHFIELD, M. S. School counselors' and school principals' perceptions of the school counselor's role. Education, v. 126, p. 10-16, 2001.

LAGO, J. R.; ONRUBIA, J. Asesoramiento psicopedagógico para la mejora de la práctica educativa. Barcelona: ICE-Horsori, 2011.

LEITHWOOD, K. ¿Cómo liderar nuestras escuelas? Aportes desde la investigación. Santiago de Chile: Fundación Chile, 2009.

MARTÍNEZ, A. C.; KRICHESKY, G. J.; GARCIA, B. El orientador escolar como agente interno de cambio. Revista Iberoamericana de Educación, v. 54, p. 107-122, 2010.

MURILlO, F. J.; KRICHESKY, G.; CASTRO, A.; HERNÁNDEZ-CASTILLA, R. Liderazgo para la inclusión escolar y la justicia social. Aportaciones de la investigación. Revista Latinoamericana de Educación Inclusiva, v. 4, n. 1, p. 169-186, 2010.

OECD. Creating Effective Teaching and Learning Environments: First Results from TALIS, 2009. Disponible en: <http://www.oecd.org/TALIS>. Acceso en: 18 ene. 2015.

OECD. Teaching and Learning International Survey TALIS 2013. Conceptual Framework. Paris: OECD Publishing, 2013.

RODRÍGUEZ, M. El asesoramiento comunitario y la reinvención del profesorado. Revista de Educación, n. 339, p. 59-75, 2006.

SANTANA, L. Los orientadores como agentes de cambio. En: BISQUERRA, R. (Ed.). Modelos de orientación e intervención psicopedagógica. Barcelona: Wolters Kluiwer, 2008. p. 121-145.

SINK, C. School Counselors as Accountability Leaders: Another Call for Action. Professional School Counseling, 2009. Disponible en: <http://www.thefreelibrary.com/>. Acceso en: 24 dic. 2015.

SPILLANE, J. P. Distributed leadership. London: Jossey-Bass, 2006.

SPILLANE, J. P.; CAMBURN, E. M.; PAREJA, A. S. Schools principals at work. A distributed perspective. En: LEITHWOOD, K.; MASCALL, B.; STRAUSS, T. Distributed leadership according to the evidence. Nueva York: Routledge, 2009. p. 87-110.

STONE C.; CLARK, M. A. School counselors and principals: Partners in support of academic achievement. NAASP Bulletin, v. 85, n. 624, p. 46-53, 2001. 
STRAUSS, A.; CORBIN, J. Bases de la investigación cualitativa. Técnicas y procedimientos para desarrollar la teoría fundamentada. Antioquia: Universidad de Antioquia, Colombia, 2002.

SWAFFIELD, S. Critical friends: supporting leadership, improving learning. Improving Schools, v. 7, n. 3, p. 267-278, 2004.

WALKER, J. Principals and counsellors working for social justice: A complementary leadership team. Guidance and Counseling, v. 21, n. 2, p. 114-124, 2006.

WERTZ, F. J.; CHARMAZ, K.; MCMULLEN, L. M. Five ways of doing qualitative analysis: Phenomenological psychology, grounded theory, discourse analysis, narrative research, and intuitive inquiry. New York: The Guilford Press, 2011.

ZEICHNER, K. M. La formación del profesorado y la lucha por la justicia social. Madrid: Morata, 2010.

Texto recebido em 16 de outubro de 2015. Texto aprovado em 16 de outubro de 2015. 